\title{
Primordial Magnetic Fields from Cosmological First Order Phase Transitions
}

\author{
Günter Sigl and Angela V. Olinto \\ Department of Astronomy and Astrophysics, Enrico Fermi Instute \\ The University of Chicago, \\ Chicago, Illinois 6063\%-1433 \\ and NASA/Fermilab Astrophysics Center \\ Fermi National Accelerator Laboratory, Batavia, IL 60510-0500 \\ Karsten Jedamzik \\ University of California, Lawrence Livermore National Laboratory \\ Livermore, CA 94550
}

(March 22, 2018)

\begin{abstract}
We give an improved estimate of primordial magnetic fields generated during cosmological first order phase transitions. We examine the charge distribution at the nucleated bubble wall and its dynamics. We consider instabilities on the bubble walls developing during the phase transition. It is found that damping of these instabilities due to viscosity and heat conductivity caused by particle diffusion can be important in the QCD phase transition, but is probably negligible in the electroweak transition. We show how such instabilities together with the surface charge densities on bubble walls excite magnetic fields within a certain range of wavelengths. We discuss how these magnetic seed fields may be amplified by MHD effects in the turbulent fluid. The strength and spectrum of the primordial magnetic field at the present time for the cases where this mechanism was operative during the electroweak or the QCD phase transition are estimated. On a $10 \mathrm{Mpc}$ comoving scale, field strengths of the order $10^{-29} \mathrm{G}$ for electroweak and $10^{-20} \mathrm{G}$ for QCD, could be attained for reasonable phase transition parameters.
\end{abstract}

PACS numbers: 98.62.En, 98.80.Cq, 12.15.Ji

\section{INTRODUCTION}

The origin of galactic and intra-cluster magnetic fields and the existence of a cosmological magnetic field are still unknown. It has been suggested that dynamo effects in turbulent fluids might amplify small seed fields exponentially [1]. However, it has been shown recently [2,3] that dynamo theory has difficulties explaining the galactic and cluster magnetic fields. Alternatively, these fields may be the result of compression of a primordial field which would then permeate the universe as a whole. In this scenario, the required strength of the primordial field on a Mpc scale would be much higher, $B_{\text {prim }} \sim 10^{-12}-10^{-9} \mathrm{G}$ at present 4 , compared to the case where the fields have been amplified by a dynamo, $B_{\text {seed }} \sim 10^{-24} \mathrm{G}$.

It is thus of some interest to investigate mechanisms which could produce primordial fields and estimate their strength and spectrum. Scenarios involving inflation can lead to large coherence lengths, but the predicted amplitude is in general very small [5.6]. Magnetic seed fields can be created by the slightly different dynamical response of the negatively and positively charged components of a quasineutral fluid such as in the Biermann bat- tery mechanism [7]. This thermoelectric effect operates as shocks form with the nucleation of bubbles during the QCD transition [8], but the created fields are also quite small. More recently, magnetic seed fields caused by the turbulent flow near the walls of bubbles which are being nucleated and grow during a cosmological first order phase transition have been estimated for the QCD [9] and the electroweak (EW) [10] transition. These two publications make different assumptions about the nature of the charge layers and the fluid flow at the bubble walls. As a result, the seed field strength estimates in Ref. [9] are larger by some 14 orders of magnitude compared to the ones in ref. [10] when each generated field is compared to the appropriate equipartition strength for each transition.

Scenarios where magnetic fields are created directly from the Higgs fields involved in the EW transition have been discussed in Refs. [11,12]. Estimates of magnetic fields generated during phase equilibration of a complex Higgs field caused by bubble collisions in a first order phase transition have been given in Ref. [13].

In this paper, we estimate the strength of primordial magnetic fields generated during a cosmological first or- 
der phase transition. We rederive the charge density distribution at the bubble wall in Sec. II. We then discuss instabilities in the bubble growth as well as their damping due to a finite shear viscosity and heat conductivity caused by particle diffusion in Sec. III. In Sec. IV, we show how the fluid flows associated with the bubble wall instabilities together with the charge density distribution at the wall will lead to the generation of magnetic seed fields. In this section we determine the wavelength range in which the magnetic fields are initially excited and the amplitude of these seed fields. In Sec. V, we discuss the transition to the non-linear regime and how the fluid turbulence can amplify the initial seed fields. We apply our scenario to the EW and the QCD transition in Sec. VI and estimate the resulting strength and spectrum of the extragalactic magnetic field at present time. A summary of our findings is contained in Sec. VII. We will use natural units and the CGS system throughout the paper.

\section{SURFACE CHARGE DENSITIES AT THE QCD AND THE EW PHASE BOUNDARIES}

An unmagnetized plasma may develop magnetic fields if the spontaneous separation of electric charges give rise to a net current. Separation of charges will occur during a first-order EW transition through the development of net baryon number or net top-quark number gradients in or at the EW phase boundaries, regardless of whether baryogenesis does or does not occur at the EW transition. During a first order QCD transition net baryon number discontinuities of appreciable magnitude may develop at the phase boundaries leading to the spontaneous separation of charges. This charge separation has been previously considered by Ref. [9] for QCD phase boundaries and by Ref. [10 for EW phase boundaries.

The first order phase transition proceeds from a hightemperature ("h") to a low-temperature ("l") phase. For the EW transition this would correspond to the phase with a vanishing and non-vanishing vacuum expectation value of the Higgs field, and for the QCD transition to the quark-gluon phase and the hadronic phase, respectively.

In the QCD transition the baryon number susceptibility in the high-temperature phase is larger than that in the low-temperature phase such that in chemical equilibrium there is a net baryon number density contrast, $R_{b}=\left(n_{b}^{h}\right)_{e q} /\left(n_{b}^{l}\right)_{e q}$, where $\left(n_{b}^{h}\right)_{e q}$ is the net baryon number for the quark-gluon phase in equilibrium (hightemperature) and $\left(n_{b}^{l}\right)_{e q}$ is the same for the hadron phase (low-temperature). $R_{b} \sim 10-10^{3}$ depending on the transition temperature. However, chemical equilibrium can only obtain for small bubble wall velocities, $v_{b} \ll 1$, and, in general, the net baryon number contrast can exceed $R_{b}$ significantly.

The width of the baryon number excess layer and the baryon number density contrast between the quark-gluon phase and the hadron phase can be estimated by studying the steady-state solutions for the net baryon density in the wall rest frame. Requiring that the net baryon flux into a thin shell in the quark-gluon phase at the phase boundary be zero, we find that

$$
v_{N}^{t h} \Sigma_{h} n_{b}^{h}\left(\frac{n_{b}^{l}}{n_{b}^{h}}-\frac{1}{R_{b}}\right)-\frac{l_{q}}{L_{b}} n_{b}^{h}+v_{b} n_{b}^{h}=0 .
$$

The first term in Eq. (11) represents the net flux of baryon number across the phase boundary where $v_{N}^{t h}$ is the thermal nucleon speed in the hadron phase and $\Sigma_{h}$ is the probability for a nucleon approaching the phase boundary from the hadron phase to dissociate into three quarks and pass over into the quark-gluon phase. The second and third terms in Eq.(11) give the diffusive baryon flux in the quark-gluon phase away from the phase boundary and the advective flux towards the phase boundary, respectively. Here $l_{q}$ is the quark mean free path and $L_{b}$ is a characteristic length scale of variation in baryon density. For large bubble velocities, $v_{b} \sim 0.1$, and/or small baryon number penetrability, $\Sigma_{h} \ll 1$ [14], the flux across the phase boundary is negligible and chemical equilibrium between the phases cannot obtain. In this case, an exponentially decaying baryon number layer with width $L_{b} \simeq l_{q} / v_{b}$ develops at the phase boundary in the quark-gluon phase while far away from the wall baryon number is homogeneously distributed. The outof-equilibrium density discontinuity at the phase boundary can then be much larger than its equilibrium value, $\left(n_{b}^{h} / n_{b}^{l}\right) \gg R_{b}$. In the opposite limit, e.g. $v_{b} \ll 1$ and/or $\Sigma_{h} \simeq 1$, equilibrium is approximately maintained such that net baryon density is roughly homogeneous in both phases. Nevertheless, there still exists a baryon number discontinuity at the phase boundary with density contrast $\left(n_{b}^{h} / n_{b}^{l}\right) \simeq R_{b}$

For the case of a first order EW transition, let us first consider the case where baryogenesis occurs at energy scales higher than the EW breaking scale. In this case a cosmic baryon number asymmetry (implying a topquark number asymmetry) already exists in the hightemperature phase through, for example, the existence of an asymmetry in (baryon - lepton) number which is conserved by baryon number violating EW sphaleron processes. Then very similar arguments to net baryon number in the QCD transition hold for net top-quark number in the EW transition. Top quark number densities are thermodynamically suppressed in the low-temperature phase due to their finite rest mass such that in equilibrium $\left(n_{t}^{h} / n_{t}^{l}\right)=R_{t} \simeq 2$. By writing a similar equation to Eq.(11) for top quarks at the EW phase boundaries, and with $v_{t o p}^{t h} \simeq 1, \Sigma_{t o p} \simeq 1$, and $R_{t}$ only slightly larger than unity, one finds that only for relativistic wall velocities, $v_{b} \simeq 1$, chemical equilibrium cannot be maintained. Since we consider $v_{b} \ll 1$ (see below), net top quark number will be in equilibrium which implies that top quark number density will vary by a factor of order unity over the extension of the EW bubble wall.

If $\mathrm{EW}$ baryogenesis is operative during a first order EW 
transition, the cosmic baryon asymmetry will be generated either in front of the wall in non-adiabatic (thin wall) baryogenesis scenarios or within the wall in adiabatic (thick wall) baryogenesis scenarios [15]. Note that for the simplest extensions of the standard model of EW interactions one expects thick walls, $L_{\text {wall }} \gg 1 / T$ [16. In this case, a baryon asymmetry (carried by $u, d, s$, $c$, and $b$ quarks) will smoothly vary from zero in the high-temperature phase to its cosmic average value deep within the wall where baryon number violating processes shut off.

In the QCD transition, the net baryon number is associated with a net positive charge density. In particular, $\rho_{c}=(2 / 3) e n_{u}-(1 / 3) e n_{d}$ in the quark-gluon phase in the limit where the strange quark mass is large compared to the temperature, and $\rho_{c}=e n_{p}$ in the hadron phase where $n_{u}, n_{d}$, and $n_{p}$ are net number densities for up-quarks, down-quarks, and protons, respectively. Globally this net positive charge is neutralized by a negative charge density due to the net electron number. However, locally on the scale of the baryon discontinuity/inhomogeneity at the phase boundary electrons are free streaming, e.g. $l_{e} \simeq\left[\left(\alpha^{2} / T^{2}\right)\left(g / \pi^{2}\right) T^{3}\right]^{-1} \gtrsim L_{b}$, such that the thin excess baryon number layer/discontinuity at the phase boundary cannot remain neutral. In the absence of electric fields, $n_{u} \simeq n_{d}$ (for $\left.n_{e} \simeq n_{\nu} \ll n_{u}\right)$, while weak equilibrium in the presence of a positive electric potential requires $n_{d}>n_{u}$ and leads to screening of the charge density by the conversion of positively charged up-quarks into negatively charged down-quarks.

Consider the grand potential density for up- and downquarks in the presence of an electric field, $E$,

$$
\Omega=-\sum_{i=u, d} \frac{21 \pi^{2} T^{4}}{180}\left[1+\frac{30}{7 \pi^{2}}\left(\frac{\mu_{i}-q_{i} \phi}{T}\right)^{2}+\ldots\right]+\frac{E^{2}}{8 \pi},
$$

where $\phi$ is the electrostatic potential, $\mu_{i}$ and $q_{i}$ are chemical potential and electric charge of species $i$, and $\Omega$ is given to lowest order in $\mu_{i}^{\prime}=\mu_{i}-q_{i} \phi$. Baryon number density $n_{b}$ and charge density $\rho_{c}$ can be obtained from the net quark number densities $n_{i}=-\left(\partial \Omega / \partial \mu_{i}\right)_{T, \phi}$, which yield

$$
\begin{aligned}
n_{b} & =\frac{1}{3} T^{2}\left(\mu_{u}^{\prime}+\mu_{d}^{\prime}\right) \\
\rho_{c} & =\frac{1}{3} e T^{2}\left(2 \mu_{u}^{\prime}-\mu_{d}^{\prime}\right) .
\end{aligned}
$$

Further demanding weak equilibrium in the case where $\mu_{e}=\mu_{\nu} \simeq 0$ (electrons and neutrinos are free streaming) gives $\mu_{d}=\mu_{u}+e \phi$. The Poisson equation together with Eq. (3) can then be used to derive the Debye screening equation

$$
\frac{\partial^{2} \phi}{\partial z^{2}}-\frac{1}{\lambda^{2}} \phi=-2 \pi e n_{b},
$$

where $\lambda=\left(4 \pi e^{2} T^{2}\right)^{-1 / 2}$ in this model. We note here that the actual Debye screening length in a multi-species relativistic plasma is $\lambda_{D} \simeq\left(4 \pi e^{2} g_{c} T^{2}\right)^{-1 / 2}$ where $g_{c}$ is the statistical weight of relativistic charged particles with mean free path $l \lesssim \max \left[\lambda_{D}, L_{b}\right]$.

The Debye equation can be solved for the expected steady state net baryon number distribution in the QCD transition and the net top-quark/baryon number distribution in the EW transition. For the QCD phase boundaries we find a surface charge density

$$
\sigma_{c} \simeq e n_{b}^{l}\left(\frac{n_{b}^{h}}{n_{b}^{l}}-1\right) \lambda_{D}+\frac{1}{2} e n_{b}^{h}\left(\frac{\lambda_{D}}{L_{b}}\right)^{2} L_{b} .
$$

Here the first term arises from a charge density on the wall (the width of the wall is of order $\sim 1 \mathrm{fm} \ll \lambda_{D}$, approximately a QCD-color screening length) and is spread over approximately one electric Debye screening length, whereas the second term results from a much smaller charge density on the scale of the baryon number inhomogeneity in the quark-gluon plasma, $L_{b} \gg \lambda_{D}$. The positive charge at the phase boundary in the quarkgluon plasma is balanced by a negative charge density at the wall in the hadron phase, resulting in a thin electric dipole layer. The baryon density at the wall in the quark-gluon phase, $n_{b}^{h} \gg n_{b}^{l}$, is approximately $n_{b}^{h} \simeq 2 \bar{n}_{b}$ halfway into the transition when chemical equilibrium is maintained, but can become appreciable towards the end of the transition, $n_{b}^{h} \gg R_{b} \bar{n}_{b}$. Here $\bar{n}_{b}$ is the cosmic average baryon density, $\bar{n}_{b} \simeq 40 \eta_{S}(g / 100) T^{3}$ with $\eta_{S} \simeq 5 \times 10^{-11}$ the cosmic average baryon-to-entropy ratio.

In the EW transition there are two separate cases depending on the width of the EW phase boundary, in particular either thick walls $L_{\text {wall }} \gg \lambda_{D}$, or thin walls $L_{\text {wall }} \lesssim \lambda_{D}$. The resulting surface charge density, if EW baryogenesis is not operative, is

$$
\begin{aligned}
& \sigma_{c} \simeq e n_{t o p}^{l}\left(\frac{n_{t o p}^{h}}{n_{t o p}^{l}}-1\right)\left(\frac{\lambda_{D}}{L_{\text {wall }}}\right)^{2} L_{\text {wall }}, \quad \text { if } L_{\text {wall }} \gg \lambda_{D} \\
& \sigma_{c} \simeq e n_{t o p}^{l}\left(\frac{n_{t o p}^{h}}{n_{t o p}^{l}}-1\right) \lambda_{D}, \quad \text { if } L_{\text {wall }} \lesssim \lambda_{D}
\end{aligned}
$$

and is seen to be suppressed by $\left(\lambda_{D} / L_{\text {wall }}\right)^{2}$ for continuous thick walls. Here $n_{\text {top }}^{h} \simeq(1 / 6) \bar{n}_{b} \gtrsim n_{\text {top }}^{l}$ for all but the largest wall velocities, $v_{b} \simeq 1$. The magnitude and spatial extension of charge densities when EW baryogenesis does occur is of the same order as that given in Eq.(6).

To summarize, we parametrize the charge density at the bubble wall by

$$
\rho_{c} \sim e \eta T_{c}^{3},
$$

which extends over a length scale $l_{c} \sim f_{c} / T_{c}$ around the wall. In the QCD case $\eta \sim 5 \times 10^{-10}-10^{-5}$, and $f_{c} \sim 1$ for the dominant electric charge density resulting 
from the baryon number discontinuity right at the phase boundary, and for the EW transition, $\eta \sim 10^{-14}$ and $f_{c} \sim 40$, where we assume thick walls, $L_{\text {Wall }} \approx 40 / T$.

\section{INSTABILITIES OF BUBBLE GROWTH AND VISCOUS DAMPING IN THE LINEAR REGIME}

During cosmological first order phase transitions, instabilities may develop as bubbles grow. Hydrodynamic instabilities can occur when the transport of latent heat is dominated by the fluid flow. These have been investigated for the QCD transition [17] and the EW transition [18] in the small velocities limit, for cosmological detonation fronts 19], and for general first order transitions in the limit of very small or very large velocities [20]. These bubble wall instabilities may be damped by finite viscosity and heat conductivity due to the diffusion of radiation on small length scales. To account for this damping we will use the approach in Refs. 221,22] for length scales larger than the radiation mean free path.

We note that, depending on the parameters of the phase transition, the heat transport may be dominated by diffusion instead of convection. In that case, bubbles larger than the radiation mean free path would likely become unstable to "dendritic growth" 23. We discuss below under which conditions this may happen.

The high and low temperature phases for either the EW or the QCD transition can be described by the following two equations of state for pressure $p_{i}(T)$, enthalpy density $w_{i}(T)$, energy density $\rho_{i}(T)(i=h, l)$, and temperature $T$ :

$$
\begin{array}{cl}
p_{l}(T)=\left[w_{l}(T)+L\right] / 4, & \rho_{l}(T)=\left[3 w_{l}(T)-L\right] / 4 \\
p_{h}(T)=w_{h}(T) / 4, & \rho_{h}(T)=3 w_{h}(T) / 4
\end{array}
$$

where $w_{i}(T)=\left(2 \pi^{2} / 45\right) g_{i} T^{4}(i=h, l)$, and $g_{i}$ is the number of relativistic degrees of freedom in the plasma. Furthermore, $\delta \equiv\left(w_{h}-w_{l}\right) / w_{h}=L / w_{h}>0$, with $w_{i} \equiv w_{i}\left(T_{c}\right)(i=h, l)$ and $L$ the latent heat. The critical temperature $T_{c}$ is defined by $p_{l}\left(T_{c}\right)=p_{h}\left(T_{c}\right)$ or, equivalently, by equating the free energy densities in the two phases. We assume that charged and strongly interacting particles have approximately the same velocity $\mathbf{v}$ and can thus be described in the one-fluid approximation. The fluid carries a conserved quantum number, namely baryon number, with a flux given by $n^{\mu}=n U^{\mu}$. Here, $n$ is the proper baryon number density and $U^{\mu} \simeq(1, \mathbf{v})$ is the four velocity (we assume non-relativistic flows).

In order to obtain the fluid flow and electromagnetic (EM) fields during bubble nucleation in a first order phase transition, one would have to solve the following equations in the presence of a phase boundary simultaneously: Energy-momentum conservation, $T^{\mu \nu} ; \nu=0$, where $T^{\mu \nu}=(\rho+p) U^{\mu} U^{\nu}-p g^{\mu \nu}+\tau^{\mu \nu}+T_{\mathrm{EM}}^{\mu \nu}$ is the energy-momentum tensor including non-ideal contributions $\tau^{\mu \nu}$ from a finite viscosity and heat conductivity caused by the diffusion of photons and neutrinos [21] as well as EM contributions $T_{\mathrm{EM}}^{\mu \nu}$; baryon number conservation, $n_{; \mu}^{\mu}=0$; and, finally, Maxwell's equations.

A full solution of this set of non-linear equations would require extensive numerical calculations. Analytical approximations are usually obtained by linearizing the equations around their zeroth order equilibrium solution. This has been done in the literature for various limiting cases: For example, in Ref. [22], the case of only one phase was considered and dispersion relations and damping rates for MHD waves were obtained. In this work, Maxwell's equations were treated in the ideal MHD approximation (assuming infinite conductivity which is an excellent approximation for the evolution of the magnetic field $\mathbf{B}$ in the plasma under consideration [9]), i.e. the hydromagnetic equation

$$
\partial_{t} \mathbf{B}=\boldsymbol{\nabla} \times(\mathbf{v} \times \mathbf{B})
$$

was used.

For an ideal fluid (i.e. vanishing viscosity and heat conductivity, $\tau^{\mu \nu}=0$ ) and no EM fields, the remaining linearized equations for the perturbations in pressure, $p^{\prime}$, and velocity, $\mathbf{v}^{\prime}$, are [17, 18]

$$
\begin{array}{r}
\left(\partial_{t}+\mathbf{v} \cdot \boldsymbol{\nabla}\right) p^{\prime}+\frac{w}{3} \boldsymbol{\nabla} \cdot \mathbf{v}^{\prime}=0 \\
\left(\partial_{t}+\mathbf{v} \cdot \boldsymbol{\nabla}\right) \mathbf{v}^{\prime}+\frac{1}{w} \boldsymbol{\nabla} p^{\prime}-\frac{1}{3} \mathbf{v} \boldsymbol{\nabla} \cdot \mathbf{v}^{\prime}=0
\end{array}
$$

and have to be solved in both phases. Both the zeroth order solutions for a planar bubble wall, represented by the unperturbed quantities $\mathbf{v}$ and $p$ (or $w$ ) and the perturbations $\mathbf{v}^{\prime}$ and $p^{\prime}$ have to be matched at the phase boundary by requiring continuity of energy and momentum flow and the transverse velocity across the interface. The latter condition comes from assuming equilibrium in the presence of a finite shear viscosity. It has been shown in Ref. [20] that growing instabilities seem to be possible only if the bubble wall velocity $v_{b}$ satisfies

$$
v_{b} \leq v_{\text {crit }} \equiv\left[\left(T_{c}^{2}-T_{h}^{2}\right) / 2 T_{c}^{2}\right]^{1 / 2},
$$

where $T_{h}$ is the temperature in the high-temperature phase. The treatment in Refs. 17, 18 is only adequate in the limit $v_{b} \ll v_{\text {crit }}$. It was also pointed out 20] that the QCD transition is a borderline case since $v_{b} \simeq$ $v_{\text {crit }} \simeq 0.03$, whereas the $\mathrm{EW}$ transition is unlikely to fulfill Eq. (11) in the initial stage of fast bubble growth. However, since $v_{b} \propto\left(T_{c}^{2}-T_{h}^{2}\right) / T_{c}^{2}$ [16], the condition Eq. (11) may eventually be satisfied if $T_{h} \rightarrow T_{c}$. Indeed, Ref. 24] argued that the EW bubble walls may slow down significantly (by $1-2$ orders of magnitude) if reheating is significant. For reasonable phase transition parameters, $v_{b} \lesssim 10^{-2}$ during this stage, and Eq. (11) can be met. It is therefore possible that hydrodynamic instabilities develop both in the QCD transition and during the late stages of the EW transition for which the approach in Refs. [17.18] can be applied. In the following, we will assume that this is indeed the case. 
Here, we are interested in solutions of the general linearized equations for two phases including viscosity and heat conductivity and EM fields. This is still a quite complicated problem and one usually considers the simpler limiting cases of either one phase of a viscous fluid or two phases of an ideal fluid. In the general case, we expect a certain wavelength range over which there are growing instability modes, i.e. the growing instability dominates over the diffusive damping. Since we are only interested in an order of magnitude estimate of the resulting initial seed fields and their coherence scale, we can restrict ourselves to the case where the damping rate is small compared to the instability growth rate. We can then describe these modes by Eq. (10) and verify a posteriori that the damping rate $\gamma_{\mathrm{d}}$ of the corresponding bubble wall instability is small compared to its growth rate $\gamma_{\text {inst }}$. Seed fields can then be generated by these growing bubble wall instabilities. We will verify that backreaction effects of these seed fields onto the fluid flow in the growing instabilities are negligible also.

Let $v_{h}$ and $v_{l}$ be the modulus of the unperturbed velocity of the high- and low-temperature phase, respectively, in the bubble wall rest frame. We assume that the first order transition proceeds as a weak, non-relativistic deflagration, i.e. $v_{h}, v_{l} \ll c_{s}$, and $\delta \simeq\left(v_{l}-v_{h}\right) / v_{h} \ll 1$, where $c_{s} \simeq 1 / \sqrt{3}$ is the speed of sound, and get [17, 18

$$
\gamma_{\mathrm{inst}} \simeq \delta v_{h} k / 2
$$

This holds true in the range of wavenumbers $k_{\min } \lesssim k \lesssim$ $k_{\max }$, with

$$
\begin{aligned}
& k_{\min }=2 / R \delta, \\
& k_{\max }=\delta w_{h} v_{h}^{2} / \sigma,
\end{aligned}
$$

Here, the first condition comes from requiring that $\gamma_{\mathrm{d}}$ be larger than the expansion rate $v_{h} / R$ of a spherical bubble of radius $R$, whereas the second marks the boundary to the regime where the bubble wall is stabilized by the surface tension, $\sigma \sim T_{c}^{3}$. The hydrodynamic instability therefore can become operative for bubbles with a radius $R$ large enough such that $k_{\min }<k_{\max }$. This is certainly the case for the fully developed bubbles near percolation which we consider in this paper. For these bubbles, $R \sim R_{\text {perc }} \simeq f_{b} r_{H}$, where $r_{H} \simeq 2.3 \times$ $10^{6}(\mathrm{~g} / 10)^{-1 / 2}(T / 100 \mathrm{MeV})^{-2} \mathrm{~cm}$ is the Hubble radius, and $g$ is the number of relativistic degrees of freedom in the plasma. For the QCD case, $f_{b} \sim 10^{-6}-10^{-2}$ [25,26], and for the EW case $f_{b} \sim 10^{-5}-10^{-4}$ 24,26].

The growth rate in Eq. (12) has to be compared with the damping rate

$$
\gamma_{\mathrm{d}} \simeq \frac{g_{t}}{5 g} \min \left[k^{2} \tau_{r}, \frac{1}{\tau_{r}}\right]
$$

where $g_{t}$ is the statistical weight for particles efficiently transporting momentum, either neutrinos $\left(g_{t}=5.5\right)$ or photons $\left(g_{t}=2\right)$, and $\tau_{r}=\left(\left\langle\sigma_{r}\right\rangle g T^{3} / \pi^{2}\right)^{-1}$ is the mean free path of these particles, and $\left\langle\sigma_{r}\right\rangle$ is the corresponding average cross section. For $k \tau_{r} \lesssim 1$, Eq. (14) was obtained from Ref. [22], and for $k \tau_{r} \gtrsim 1$, the damping rate is approximately independent of $k$ and proportional to $1 / \tau_{r}$ 27]. Associated with the damping rate of instabilities is the Reynolds number of the fluid flow at length scale $1 / k$,

$$
R(k) \equiv \frac{v_{f}(k)}{k \tau_{r}}
$$

where $v_{f}(k)$ is the typical fluid velocity at that length scale. In the linear regime of perturbations, $v_{f}(k) \lesssim v_{h}$.

For the EW transition, the dominant processes governing diffusion of radiation turn out to be EM bremsstrahlung, $e+l \rightarrow e+l+\gamma$ and photon-electron pair production, $e+\gamma \rightarrow e+l^{+} l^{-}$, where $l$ stands for any charged lepton in the plasma. These processes are influenced by plasma effects. With $\alpha$ the fine structure constant, $n_{c}$ the density of charged particles in the plasma, and $m_{p} \simeq\left(4 \pi \alpha n_{c} / 3 T\right)^{1 / 2} \simeq 0.5 T(g / 100)^{1 / 2}$ the plasma mass of the electron at temperature $T \gg 1 \mathrm{MeV}$ [28], the relevant cross sections can be written as $\left\langle\sigma_{r}\right\rangle=\left\langle\sigma_{\mathrm{EM}}\right\rangle \simeq$ $\left(8 \alpha^{3} / m_{p}^{2}\right) \ln \left(6.3 T / m_{p}\right) \simeq 3 \times 10^{-5}(g / 100)^{-1} / T^{2}$. This leads to the Reynolds number for EM viscosity,

$$
\begin{aligned}
R_{\mathrm{EM}}(k) \simeq & 4 \times 10^{11}\left(\frac{g}{100}\right)^{-1 / 2}\left(\frac{T_{c}}{100 \mathrm{GeV}}\right)^{-1} \\
& \times f_{b} \delta v_{f}(k) \frac{k_{\mathrm{min}}}{k}
\end{aligned}
$$

where $m_{\mathrm{Pl}}$ is the Planck mass. Using the numerical parameters $f_{b} \gtrsim$ a few $\times 10^{-5}, \delta \sim 10^{-3}$, and $v_{h} \simeq v_{b}=$ $v_{l} \sim 0.01$ in the percolation stage of the EW transition [24, 26, 29, we get $R_{\mathrm{EM}}\left(k_{\min }\right) \gtrsim 10^{2}$.

For the QCD transition, the damping rate Eq. (14) is dominated by neutrino diffusion for $k \lesssim\left(\tau_{\mathrm{EM}} \tau_{\nu}\right)^{-1 / 2}$, where $\tau_{\mathrm{EM}}$ and $\tau_{\nu}$ are the mean free paths of EM radiation and neutrinos, respectively. Using $\left\langle\sigma_{r}\right\rangle=\left\langle\sigma_{\nu}\right\rangle \simeq$ $2.1 G_{\mathrm{F}}^{2} T^{2}$, where $G_{\mathrm{F}}$ is Fermi's constant, we obtain for the Reynolds number for neutrino viscosity

$$
R_{\nu}(k) \simeq 10^{5}\left(\frac{g}{10}\right)^{1 / 2}\left(\frac{T_{c}}{100 \mathrm{MeV}}\right)^{3} f_{b} \delta v_{f}(k) \frac{k_{\min }}{k}
$$

Using the parameters $f_{b} \sim 10^{-2}, \delta \sim 10^{-1}$, and $v_{h} \sim$ 0.129 for the QCD transition, we get $R_{\nu}\left(k_{\min }\right) \sim 1$ and $R_{\mathrm{EM}}\left(k_{\mathrm{min}}\right) \sim 10^{12}$.

Combining Eqs. (12) and (14), we obtain

$$
\frac{\gamma_{\mathrm{d}}}{\gamma_{\mathrm{inst}}} \simeq \frac{0.2}{\delta v_{h}}\left(\frac{g}{10}\right)^{-1}\left(\frac{g_{t}}{5.5}\right) \min \left[k \tau_{r},\left(k \tau_{r}\right)^{-1}\right],
$$

and instabilities are expected to be damped in the wavenumber range $5(g / 10)\left(g_{t} / 5.5\right)^{-1} R\left(k_{\min }\right) \lesssim$ $k / k_{\min } \lesssim 0.2(g / 10)^{-1}\left(g_{t} / 5.5\right) R\left(k_{\min }\right) /\left(\delta v_{h}\right)^{2}$. From this we see immediately, that, at least in case of the EW transition, damping is unlikely to be important at the largest length scales over which instabilities can develop, 
$k \sim k_{\text {min }}$. Damping probably only plays a role on length scales which are a few orders of magnitude smaller than $1 / k_{\min }$, in a window of relatively small logarithmic width of $\sim 2 \log \left[\left(g_{t} / 5.5\right) / \delta v_{h}(g / 10)\right]-1.4$. In contrast, for the QCD transition, damping could become important around $k_{\min }$, if $R_{\nu}\left(k_{\min }\right) \lesssim 1$.

We note that since $\gamma_{\text {inst }}\left(k_{\text {min }}\right)=v_{h} / R$ is roughly the convection rate, $\gamma_{\mathrm{d}}\left(k_{\min }\right) / \gamma_{\text {inst }}\left(k_{\min }\right) \gtrsim 1$ corresponds to the case where transport of latent heat is dominated by diffusion instead of hydrodynamic flow. Therefore, depending on the exact phase transition parameters entering Eqs. (16), (17), and (18), this situation could occur in the QCD transition, as remarked in Ref. [23], but not for the EW transition.

\section{SEED FIELDS FROM HYDRODYNAMIC INSTABILITIES}

Seed fields associated with instabilities will be generated when positive and negative charges are displaced relative to each other and, therefore, the one fluid approximation cannot be used. However, the resulting EM currents, $j \sim \rho_{c} v \sim e \eta T_{c}^{3} v$, correspond to very small conduction velocities, $v_{c}$, of negative relative to positive charge carriers, $v_{c} \sim \eta v \ll v$. Therefore, the subsequent evolution of the seed fields due to dynamo effects can very well be described within the MHD approximation. Note that seed fields are necessary since the MHD equation (9) is homogeneous in B. We now estimate the seed fields generated by the hydrodynamic instabilities.

Let the planar, undisturbed phase boundary be located at $z=0$ with the low-temperature phase at $z<0$ and the high-temperature phase at $z>0$. We can choose the instability to have a wavevector in the $\mathrm{x}$ direction and the perturbation of the wall can be described by $z_{w}(x, t)=z_{0} \exp \left(\gamma_{\text {inst }} t+i k x\right)$. This corresponds to a nonpropagating instability with $\operatorname{Im} \gamma_{\text {inst }}=0$. To lowest non-trivial order in $v_{h}$ and $\delta$, and for $k \ll k_{\max }$, the solutions of Eq. (10) take a relatively simple form:

$$
\begin{aligned}
\mathbf{v}_{h}^{\prime} & =\delta v_{h}\left(\mathbf{e}_{z}-i \mathbf{e}_{x}\right) k z_{w}(x, t) e^{-k z} / 2, \\
p_{h}^{\prime} & =-\delta w_{h} v_{h}^{2} k z_{w}(x, t) e^{-k z} / 2, \\
\mathbf{v}_{l}^{\prime} & =\delta v_{h}\left[\mathbf{e}_{z} e^{\delta k z / 2}+\left(\mathbf{e}_{z}+i \mathbf{e}_{x}\right) e^{k z}\right] k z_{w}(x, t) / 2, \\
p_{l}^{\prime} & =-\delta w_{h} v_{h}^{2} k z_{w}(x, t) e^{k z} / 2,
\end{aligned}
$$

where $\mathbf{e}_{x}$ and $\mathbf{e}_{z}$ are unit vectors in the $x$ and $z$ direction, respectively. Note that Eq. (19) only holds for distances from the wall which are much larger than the perturbation amplitude of the wall location, i.e. $z \gg z_{w}(x, t)$. It is seen from Eq. (19) that the instability perturbs the fluid flow in the low-temperature phase up to a characteristic distance $1 / \delta k$, whereas in the high-temperature phase the fluid flow is only perturbed up to a distance $1 / k$. For the wavelength regime under consideration, Eq. (13), these scales are typically much larger than the width $\sim f_{c} / T_{c}$ of the dipole layer.
Eq. (19) illustrates that, for a given mode with $\mathbf{k}=$ $k \mathbf{e}_{x}$, the $x$ component of the perturbed velocity field has a discontinuity at $z=0$ :

$$
\left(\mathbf{v}_{h}^{\prime}-\mathbf{v}_{l}^{\prime}\right) \cdot \mathbf{e}_{x}=-i \delta v_{h} k z_{w}(x, t) .
$$

The dipole layer with charge density given by Eq. (7) thus leads to a net current in the $x$ direction,

$$
j_{x} \sim e \eta T_{c}^{3} \delta v_{h} k z_{w}(x, t) e^{-T_{c}|z| / f_{c}},
$$

provided $f_{c} / T_{c} \gtrsim z_{w}(x, t)$. We can now superpose all instability modes from Eq. (19) with $\mathbf{k}$ in the $z=0$ plane and write the wall displacement spectrum as $\left\langle z_{w}^{2}\right\rangle=$ $\int\left[z_{w}(k)\right]^{2} d \ln k$. Using Ampére's law without the displacement current, $\boldsymbol{\nabla} \times \mathbf{B}=4 \pi \mathbf{j}$, and Eq. (21), after performing a three dimensional Fourier transformation, we arrive at

$$
B_{s}(k) \sim 4 e \eta T_{c}^{2} \delta v_{h} f_{c}\left(k_{\min } k\right)^{1 / 2}\left|z_{w}(k)\right|
$$

for the seed field spectrum $\left\langle B_{s}^{2}\right\rangle=\int\left[B_{s}(k)\right]^{2} d \ln k$. Strictly speaking, Eq. (22) is only reliable for $z_{w}(k) \lesssim$ $f_{c} / T_{c}$.

The instabilities Eq. 19 grow non-linear when the perturbed velocity spectrum

$$
v_{f}(k) \sim \delta^{1 / 2} v_{h}\left(k_{\min } k\right)^{1 / 2}\left|z_{w}(k)\right|
$$

satisfies $v_{f}(k) \gtrsim v_{h}$, where, analogous to the seed field spectrum, the flow velocity spectrum is written as $\left\langle v_{f}^{2}\right\rangle=\int\left[v_{f}(k)\right]^{2} d \ln k$. Furthermore, the seed field $\mathbf{B}_{s}$ and its source currents $\mathbf{j}$ give rise to an additional force acting onto the fluid of the form $\mathbf{j} \times \mathbf{B}$. This term is of second order in the perturbation amplitude and was neglected in Eq. (10). We now compare its magnitude with the leading force term $\nabla p^{\prime}$ which is of first order in the perturbation. By using Eqs. (19), Ampére's law and an equation analogous to Eq. (23) for $\left|\nabla p^{\prime}\right|$, we obtain

$$
\frac{\left|j B_{s}\right|}{\left|\nabla p^{\prime}\right|} \simeq \frac{\left(e \eta f_{c}\right)^{2}}{v_{h}} \frac{T_{c}^{4}}{w_{h}} \delta^{1 / 2} v_{f}(k)
$$

on a length scale $1 / k$. This is small compared to unity in the linear regime where $v_{f}(k) \lesssim v_{h}$. In addition, the electric field $E_{s}(k)$ induced by the growing magnetic seed field, $E_{s}(k) \sim\left[\partial B_{s}(k) / \partial t\right] / k \sim \delta v_{h} B_{s}(k)$, is small compared to the zeroth order field $E \sim e \eta f_{c} T_{c}^{2}$ caused by the electric charge layer, $E_{s}(k) / E \sim v_{f}(k) \ll 1$. We therefore conclude that in most cases backreaction effects of the seed fields onto the medium are negligible in the linear regime. Therefore, a conservative estimate of the seed field $B_{s}$ is given by assuming $z_{w}(k) \sim f_{c} / T_{c}$;

$$
B_{s}(k) \sim 4 e \eta f_{c} T_{c}^{2} \delta^{1 / 2} v_{f}(k) \gtrsim 4 e \eta T_{c}^{2} \delta v_{h} f_{c}^{2}\left(\frac{k_{\min } k}{T_{c}^{2}}\right)^{1 / 2} .
$$


Being less conservative, one can extrapolate the first expression in Eq. (25) up to the transition to the non-linear regime, where $v_{f}(k) \simeq v_{h}$, and obtain the optimistic estimate $B_{s}(k) \sim e \eta T_{c}^{2} \delta^{1 / 2} v_{h} f_{c}$. In the next section we will show that once the non-linear regime is reached, enough turbulence in the fluid is expected to build up and the seed fields can be amplified by dynamo effects.

For comparison, the thermoelectric effect discussed in Ref. [8] for the QCD transition leads to seed fields of the order $\sim 0.01 v_{f} T_{c} / R_{\text {perc }}$ which is smaller than Eq. (25) for $k \gg k_{\min }$. This can be seen by rewriting Eq. (25) as $B_{s}(k) \gtrsim 4 \eta v_{h} f_{c}^{2}\left(T_{c} / R_{\text {perc }}\right)\left(k / k_{\text {min }}\right)^{1 / 2}$. Other mechanisms have been investigated in the literature starting from thermal field fluctuations on a length scale $\simeq 1 / T_{c}$, $B_{t h}^{2}\left(k \simeq T_{c}\right) \simeq 8 \pi T_{c}^{4}$. On larger scales, $k<T_{c}$, the magnetic field spectrum was argued to behave as a power-law, $B_{t h}(k) \simeq(8 \pi)^{1 / 2} T_{c}^{2}\left(k / T_{c}\right)^{p}$. Several options have been discussed for $p$ : Based on stochastic arguments applied to the order parameter involved in the phase transition, Ref. [11] argued for $p=1$, and Ref. [12] for $p=1 / 2$. In contrast, treating the resulting magnetic dipole moments as stochastic variables leads to $p=3 / 2$ [31. In any case, by comparing with Eq. (25) we see that for $p \gtrsim 1$, very roughly, on a scale $k \simeq k_{\min }$ given by Eq. (13), our mechanism leads to comparable or stronger fields already in the linear regime.

\section{AMPLIFICATION OF SEED FIELDS BY MHD EFFECTS}

The seed fields discussed in the previous section can be amplified by exchange of energy with the turbulent fluid flow. This non-linear MHD regime is usually investigated by numerical simulations [32]. The magnetic field energy $E_{M}$ typically grows up to equipartition with the fluid flow where it takes the value

$$
E_{M}^{e q}=\frac{B_{e q}^{2}}{8 \pi}=g T^{4} v_{f}^{2},
$$

with $B_{e q}$ being the corresponding equipartition field. The fluid velocity in the turbulent regime can be estimated by assuming that the latent heat release $L \simeq \delta w_{h}$ is at least partially converted into turbulent motion. This results in $v_{f} \simeq \delta^{1 / 2}$ which is probably somewhat larger than $v_{f} \lesssim v_{h}$ in the linear regime.

We note in passing that the resulting strong equipartition fields $B_{e q}$ on bubble scales do not cause a problem with recent bounds from big-bang nucleosynthesis [33]. This is because fields on scales smaller than the comoving horizon size at neutrino-decoupling are expected to be significantly damped by neutrino diffusion by the time nucleosynthesis commences 22].

The required amount of amplification for the seed field in Eq. (25) is given by the inverse of the ratio

$$
\frac{B_{s}(k)}{B_{e q}} \simeq 0.1 \eta f_{c} v_{f}(k)\left(\frac{g}{10}\right)^{-1 / 2}
$$

$$
\gtrsim 10^{-17}\left(\frac{T_{c}}{100 \mathrm{GeV}}\right) \frac{\eta f_{c}^{2}}{f_{b} \delta^{1 / 2}} v_{h},
$$

which is $\gtrsim 10^{-24}\left(\eta / 10^{-14}\right)$ for the $\mathrm{EW}$ and $\gtrsim$ $10^{-24}\left(\eta / 10^{-5}\right)$ for the QCD transition, for the parameters used above. Note, that in case of the EW transition, $B_{s} / B_{e q}$ is much larger than what was estimated in Ref. 10] if it is scaled to the same $\eta$. The reason is that our instability analysis revealed the existence of a monopole layer of an EM current on the bubble wall, whereas in Ref. 10] a dipole layer was assumed.

The central question in the theory of magnetic field amplification in a turbulent fluid concerns the time scale over which energy equipartition is achieved and the spectrum of the resulting magnetic field. Here we use the analytic approach and physical arguments developed in Ref. [3] which was recently applied to the problem of galactic field generation [34]. Once the bubbles start to collide, vorticity is expected to develop which, on a scale $k$ is of the order $k v^{\prime}(k)$ and corresponds to a turnover rate of equal size. This turnover rate becomes larger than the instability growth rate Eq. (12) once $v^{\prime}(k) \gtrsim \delta v_{h}$. If the Reynolds number $R(k) \gg 1$, one therefore expects the fluid flow to turn turbulent before the instabilities become non-linear, which occurs for $v^{\prime}(k) \simeq v_{h}$. At that point, as long as the velocities involved are smalled compared to the speed of sound $c_{s} \simeq 1 / \sqrt{3}$, one expects a Kolmogorov type of spectrum to develop. The typical velocity on a scale $k$ is then given by $v_{f}(k) \simeq v_{f}\left(k_{c} / k\right)^{1 / 3}$ [35]. This holds for $k_{c} \lesssim k \lesssim k_{\mathrm{vis}} \simeq k_{c} R\left(k_{c}\right)^{3 / 4}$, where $k_{c}$ is the wavenumber for which the eddy turnover rate $\simeq k_{c} v_{f}$ is equal to the inverse of the percolation time $t_{\text {perc }} \simeq R_{\text {perc }} / v_{h}$, giving $k_{c} \simeq v_{h} / R_{\text {perc }} v_{f} \simeq 1 / R_{\text {perc }}$. The viscous scale $k_{\text {vis }}$, at which dissipation becomes important, is basically the scale of the smallest eddy in the turbulent flow.

Neglecting resistive damping for the moment, the growth rate of the magnetic field energy during the MHD regime is given by [3]

$$
\frac{d E_{M}}{d t}=2 \gamma_{M} E_{M}
$$

with

$$
2 \gamma_{M} \simeq \int v_{f}(k) d k
$$

where the velocity spectrum is again written as $\left\langle v_{f}^{2}\right\rangle=$ $\int\left[v_{f}(k)\right]^{2} d \ln k$. Thus, $\gamma_{M}$ is basically the maximum turnover rate of the eddies in the flow. For a Kolmogorov spectrum,

$$
\gamma_{M} \simeq v_{f} k_{c}\left(\frac{k_{\mathrm{vis}}}{k_{c}}\right)^{2 / 3} \simeq v_{f} k_{c}\left[R\left(k_{c}\right)\right]^{1 / 2} .
$$

The resistive scale $k_{r}$ is defined as the wavenumber where the resistive damping rate $\simeq-k^{2} /\left(4 \pi \sigma_{\text {cond }}\right)$ 
caused by the finite conductivity $\sigma_{\text {cond }} \simeq 10 T_{c}$ becomes comparable to the growth rate $2 \gamma_{M}$. Thus, $k_{r} \simeq\left(4 \pi \sigma_{\text {cond }} \gamma_{M}\right)^{1 / 2} \simeq k_{\text {vis }}\left[R_{M} / R\left(k_{c}\right)\right]^{1 / 2}$, where $R_{M}=$ $4 \pi\left(2 \pi / k_{c}\right) v_{f} \sigma_{\text {cond }} \simeq 10^{5}(g / 100)^{-1} R_{\mathrm{EM}}\left(k_{c}\right)$ is the magnetic Reynolds number. The magnetic field spectrum cuts off exponentially for $k \gtrsim k_{r}$. It has been shown in Ref. [34] that $\gamma_{M}$ is constant and $E_{M}$ grows exponentially until $2 \gamma_{M} E_{M}$ becomes comparable with the turbulent power $P \simeq g T_{c}^{4} \int v_{f}^{3}(k) d k \simeq g T_{c}^{4} k_{c} v_{f}^{3} \ln R\left(k_{c}\right)$ which is also the dissipation rate due to viscosity. At that point, the Alfvén velocity $v_{A} \simeq\left(2 E_{M} / g T_{c}^{4}\right)^{1 / 2}$ becomes comparable to the turbulent velocity at the viscous scale, $v_{f}\left(k_{\mathrm{vis}}\right) \simeq v_{f} R\left(k_{c}\right)^{-1 / 4}$, which implies $E_{M} \simeq$ $E_{M}^{e q} R\left(k_{c}\right)^{-1 / 2}$. Since the magnetic field on scales $k$ with $k v_{A} \gtrsim \gamma_{M} \simeq k_{\text {vis }} v_{f}\left(k_{\mathrm{vis}}\right)$ cannot grow, the magnetic field energy should be concentrated around $k_{\text {vis }}$ by the time when $P \simeq 2 \gamma_{M} E_{M}$. From then on, $2 \gamma_{M} E_{M} \simeq P$ is roughly constant, so that $E_{M}$ only grows linearly in time until it reaches $E_{M}^{e q}$. Therefore, at the same time, $\gamma_{M}$ has to decrease until it reaches $\simeq v_{f} k_{c}$. By comparing with the second equality in Eq. (30), this means that the high wavenumber cutoff in the velocity power spectrum decreases until it reaches $k_{c}$. The viscous length scale grows due to the extra viscosity caused by the energy drain from the smallest eddy to the magnetic field. The same happens to the magnetic field spectrum, so that in the end all the magnetic energy $E_{M}^{e q}$ is concentrated at $k=k_{c}$, i.e. at the largest wavelengths. The time scale over which this process occurs can be estimated as

$$
t_{M} \simeq \int \frac{d E_{M}}{2 \gamma_{M} E_{M}} \simeq \frac{E_{M}^{e q}}{P} \simeq \frac{1}{k_{c} v_{f}} \simeq t_{\text {perc }} .
$$

We have thus verified that amplification up to equipartition is possible within the percolation time. Furthermore, the magnetic field is expected to be coherent on the scale $k_{c}$, and we can write $B\left(1 / k_{c}, T_{c}\right) \simeq(8 \pi g \delta)^{1 / 2} T_{c}^{2}$.

\section{RELIC EXTRAGALACTIC MAGNETIC FIELDS}

On length scales $r \gtrsim 1 / k_{c}$ we will again assume a power-law behavior $\propto\left(k_{c} r\right)^{-p}$ for the magnetic field. Since $k_{c}$ is a macroscopic scale compared to the correlation length of the order parameter of the phase transition which is $\sim 1 / T_{c}$, the large scale fields should be determined by the randomly oriented magnetic dipole moments, leading to $p \simeq 3 / 2$ [31, 9, 10]. For the magnetic field on a comoving scale $r$ at redshift $z$ this eventually leads to

$$
\begin{aligned}
B(r, z) \sim & 10^{-21}(1+z)^{2} f_{b}^{3 / 2} \delta^{1 / 2}\left(\frac{g}{100}\right)^{-1 / 4} \\
& \times\left(\frac{T_{c}}{100 \mathrm{GeV}}\right)^{-3 / 2}\left(\frac{r}{10 \mathrm{Mpc}}\right)^{-3 / 2} \mathrm{G} .
\end{aligned}
$$

We could have followed previous authors by choosing a fiducial value for $r$ of $1 \mathrm{Mpc}$, which corresponds to the scale that collapsed to form a galaxy. The estimated field would then be appropriate for seeding the galactic fields. However, it was recently suggested that magnetic fields on scales smaller than the Silk scale $\simeq 10 \mathrm{Mpc}$ are likely to be damped by photon diffusion at recombination [22]. Thus, Eq. (32) probably only applies for $r \gtrsim 10 \mathrm{Mpc}$ and leads to an extragalactic magnetic field spectrum $B(r, z=0) \sim 10^{-29}(r / 10 \mathrm{Mpc})^{-3 / 2} \mathrm{G}$ for the EW transition, and $B(r, z=0) \sim 10^{-20}(r / 10 \mathrm{Mpc})^{-3 / 2} \mathrm{G}$ for the QCD transition for the parameters used above. In the latter case our mechanism thus predicts a field strength on a $10 \mathrm{Mpc}$ scale which is roughly comparable to the field expected from the Biermann battery mechanism acting on intergalactic distance scales during large-scale structure formation [34].

For comparison, the stochastic models mentioned in Sec. III lead to a field strength $B(r, z) \simeq(8 \pi)^{1 / 2} T_{0}^{2}(1+$ $z)^{2}\left(r T_{0}\right)^{-p}$, where $T_{0} \simeq 2.7 \mathrm{~K}$ is the present temperature of the cosmic microwave background. For both the EW and the QCD transition this gives $B(10 \mathrm{Mpc}, z=0) \sim$ $10^{-19} \mathrm{G}$ and $\sim 10^{-32} \mathrm{G}$ for $p=1 / 2$ [12 and $p=1$ [1], respectively.

In Refs. 37, 38] it was pointed out that hydromagnetic turbulence in the early universe might shift a given magnetic field power spectrum near equipartition with the fluid to length scales larger by a factor $f$ which can be as large as a few orders of magnitude. In that case, the length scale $r$ in the above estimates should be substituted by $r / f$. At large scales characterized by a given $r$, this could increase the fields by a few orders of magnitude.

\section{CONCLUSIONS}

We have discussed the generation of magnetic fields during the growth of weak deflagration bubbles nucleated in a cosmological first order phase transition in some detail. This proceeds essentially in two steps: First, instabilities can arise which can be described by linear perturbation theory. Together with a finite charge density along the bubble walls these instabilities cause EM currents and thus magnetic seed fields. While currently we are unable to decide unambiguously whether such instabilities form, for reasonable phase transition parameters this is at least plausible. Taking into account damping due to a finite viscosity and heat conductivity caused by radiation diffusion we showed that the resulting bubble instabilities can grow on length scales somewhat smaller than the bubble radius at percolation. Once these perturbations grow non-linear, the fluid is expected to turn turbulent and the seed fields can be amplified by MHD effects in the form of a dynamo. Qualitative physical arguments show that equipartition of the magnetic field energy with the kinetic energy in the turbulent motion can be achieved and the field spectrum can be concentrated at length scales not much smaller than the bubble 
radius at percolation. Random superposition of the magnetic dipole moments associated with the bubbles leads to large scale fields which can be estimated and have a characteristic spectrum. On a $10 \mathrm{Mpc}$ comoving scale, field strengths of the order $10^{-29} \mathrm{G}$ from the EW transition and $10^{-20} \mathrm{G}$ from the QCD transition could be attained at $z=0$ for reasonable phase transition parameters. Finally, hydromagnetic turbulence after the phase transition may further enhance the large scale fields.

\section{ACKNOWLEDGMENTS}

We are indebted to Gordon Baym, Kari Enqvist, Michal Hanasz, Russell Kulsrud, Harald Lesch, Robert Rosner, and Tanmay Vachaspati for many interesting and helpful discussions on the subject of cosmological magnetic fields. This work was supported by the DoE, NSF, and NASA at the University of Chicago, by the DoE and by NASA through grant NAG 5-2788 at Fermilab, and by the Alexander-von-Humboldt Foundation. We thank the Aspen Center for Physics for the hospitality while some of this work was completed.

[1] See, e.g., E. N. Parker, Cosmical Magnetic Fields (Clarendon, Oxford, England, 1979).

[2] S. I. Vainshtein and R. Rosner, Astrophys. J. 376, 199 (1991).

[3] R. M. Kulsrud, and S. W. Anderson, Astrophys. J, 396, 606 (1992).

[4] R. M. Kulsrud, S. Cowley, A. Gruzinov, and R. Sudan, to appear in Phys. Rep.

[5] M. S. Turner and L. M. Widrow, Phys. Rev. D 30, 2743 (1988).

[6] B. Ratra, Astrophys. J. 391, L1 (1993).

[7] L. Biermann, Zs. Naturforsch. A 5, 65 (1950).

[8] J. M. Quashnock, A. Loeb, and D. N. Spergel, Astrophys. J. 344, L49 (1989).

[9] B. Cheng and A. V. Olinto, Phys. Rev. D 50, 2421 (1994).

[10] G. Baym, D. Bödeker, and L. McLerran, Phys. Rev. D 53, 662 (1996).

[11] T. Vachaspati, Phys. Lett. B 265, 258 (1991).

[12] K. Enqvist and P. Olesen, Phys. Lett. B 319, 178 (1993).

[13] T. W. B. Kibble and A. Vilenkin, Phys. Rev. D 52, 679 (1995).

[14] K. Jedamzik and G. M. Fuller, Nucl. Phys. B, B441, 215 (1995).

[15] for a review see; A. G. Cohen, D. B. Kaplan, and A. E. Nelson, Ann. Rev. Nucl. Part. Sci. 43, 27 (1993).

[16] M. Dine, R. G. Leigh, P. Huet, A. Linde, and D. Linde, Phys. Rev. D 46, 550 (1992).

[17] B. Link, Phys. Rev. Lett. 68, 2425 (1992).

[18] M. Kamionkowski and K. Freese, Phys. Rev. Lett. 69, 2743 (1992).
[19] M. Abney, Phys. Rev. D 49, 1777 (1994); L. Rezzolla, Phys. Rev. D 54, 1345 (1996).

[20] P. Huet, K. Kajantie, R. G. Leigh, B. H. Liu, and L. McLerran, Phys. Rev. D 48, 2477 (1993).

[21] S. Weinberg, Gravitation and Cosmology (New York: John Wiley \& Sons, 1972), p. 53; Astrophys. J. 168, 175 (1971).

[22] K. Jedamzik, V. Katalinić, and A. V. Olinto, Report No. astro-ph/9606080 (unpublished).

[23] K. Freese and F. C. Adams, Phys. Rev. D 41, 2449 (1990).

[24] A. F. Heckler, Phys. Rev. D 51, 405 (1995).

[25] G. M. Fuller, G. J. Mathews, and C. R. Alcock, Phys. Rev. D 37, 1380 (1988).

[26] K. Enqvist, J. Ignatius, K. Kajantie, and K. Rummukainen, Phys. Rev. D 45, 3415 (1992).

[27] see, e.g., K. Jedamzik and G. M. Fuller, Astrophys. J. 423, 33 (1994).

[28] see, e.g., J. I. Kapusta, Finite Temperature Field Theory (Cambridge University Press, Cambridge, 1989).

[29] J. Ignatius, K. Kajantie, H. Kurki-Suonio, and M. Laine, Phys. Rev. D 49, 3854 (1994).

[30] see, e.g., J. D. Jackson, Classical Electrodynamics, 2nd ed. (John Wiley \& Sons, 1962).

[31] C. J. Hogan, Phys. Rev. Lett. 51, 1488 (1983).

[32] see, e.g., F. Cattaneo, E. Kim, M. Proctor, and L. Tao, Phys. Rev. Lett. 75, 1522 (1995); C. Reyl, T. M. Antonsen, and E. Ott, Phys. Rev. Lett. 76, 2270 (1996).

[33] B. Cheng, D. N. Schramm, and J. W. Truran, Phys. Rev. D 49, 5006 (1993); D. Grasso and H. R. Rubinstein, Astropart. Phys. 3, 95 (1993); P. J. Kernan, G. D. Starkman, and T. Vachaspati, Report No. astro-ph/9509126 (unpublished); B. Cheng, A. V. Olinto, D. N. Schramm, and J. W. Truran, Report No. astro-ph/9606163 (unpublished).

[34] R. M. Kulsrud, R. Cen, J. P. Ostriker, and D. Ryu, Report No. astro-ph/9607141 (unpublished).

[35] see, e.g., L. D. Landau and E. M. Lifshitz, Fluid Mechanics, 2nd ed. (Pergamon Press, New York, 1987).

[36] J. Ahonen and K. Enqvist, Phys. Lett. B 382, 40 (1996).

[37] A. Brandenburg, K. Enqvist, and P. Olesen, Phys. Rev. D 54, 1291 (1996); P. Olesen, Report No. astro-ph/9610154 (unpublished).

[38] K. Dimopoulos and A-C. Davis, Report No. DAMTP-9617, astro-ph/9610013 (unpublished). 